\title{
LES JEUNES ET INTERNET : QUELLES REPRÉSENTATIONS, QUELS USAGES ET QUELLES APPROPRIATIONS EN 2000?
}

\section{Isabelle Bréda ${ }^{1}$}

\begin{abstract}
What do we precisely know about the relation that the young people maintain with Internet : how do they see this new tool ? What do they really know of it ? Which uses in do they have, in families or schools ? Do they feel difficulties, fascination facing Internet? Are the behaviours and representations identical if the young person is familiar of Internet or if he never used it before?

In view to bring some lighting on these questions, CLEMI took part to a research in 2000, jointly in seven countries - Belgium, Spain, France, Italy, Portugal, Quebec and Switzerland.

This study reflects three dimensions of the relation between the young and the Internet: their representations, their uses and their appropriation of this new media. It offers the photograph of a situation that has already strongly changed, of an emerging phenomenon that, far from being fleeting, seems called to invest the sociocultural landscape in a durable and determining manner. And it is doubtless one of its interests today.
\end{abstract}

Que sait-on précisément de la relation que les jeunes entretiennent avec Internet : comment voient-ils ce nouvel outil ? Que savent-ils véritablement de lui ? quels usages en ont-ils, dans la famille, à l'école ? Eprouvent-ils des difficultés, de la fascination face à Internet? Les

Recherches en communication, $\mathrm{n}^{\circ} 22$ (2004). 
comportements et les représentations sont-ils identiques lorsque les jeunes sont familiers d'Internet et lorsqu'ils ne l'ont jamais utilisé ?C'est pour apporter quelques éclairages sur ces questions qu'une recherche a été menée en 2000, conjointement dans sept pays - la Belgique, l'Espagne, la France, l'Italie, le Portugal, le Québec et la Suisse.

A l'époque où l'enquête a été menée (décembre 1999 - mai 2000), Internet était encore peu installé dans les pratiques des jeunes. Nous étions alors dans une phase de mise en place, de construction. Les ordinateurs connectés étaient encore rares dans les foyers européens, alors que le Québec se démarquait fortement avec un taux élevé en matière d'équipement familial. A l'école, ils étaient souvent réservés aux enseignants et au personnel d'encadrement des établissements, les élèves n'en ayant qu'un usage très limité en terme d'accès, de temps et de pratiques. Si l'appropriation d'Internet par les jeunes était balbutiante dans les pays européens, si les usages commençaient à se diversifier, avec un temps d'avance au Québec, en revanche jeunes européens comme jeunes québécois s'étaient déjà forgé une image d'Internet assez précise, et souvent convergente.

Cette étude reflète ces trois dimensions - représentations, usages et appropriation - et offre la photographie d'une situation qui s'est déjà fortement modifiée, celle d'un phénomène émergent qui, loin d'être passager, semble appelé à investir le paysage socioculturel de façon durable et déterminante. Et c'est là sans doute un de ses intérêts aujourd'hui.

Elle a permis de poser des jalons, de fournir des données quantitatives, mais surtout qualitatives permettant d'appréhender la relation que les jeunes tissaient, ou ne tissaient pas, avec Internet. Elle a apporté aux parents, aux responsables éducatifs et aux enseignants des pistes de réflexion pour construire les premiers parcours éducatifs autour des usages d'Internet et de la compréhension de ce média, s'appuyant sur ce que les jeunes pensaient d'Internet et sur leur manière de l'utiliser.

Aujourd'hui, elle pourrait en quelque sorte servir de rétroviseur pour mieux appréhender les évolutions, analyser plus finement dans quelle mesure les changements technologiques récents ont modifié les représentations et les usages d'Internet chez les jeunes, et à quelles conditions ils favorisent son appropriation. 


\section{Conditions de la recherche}

La recherche "Les jeunes et Internet" a été menée entre novembre 1999 et mai 2000. Elle a été dirigée par une équipe québécoise et menée dans 6 autres pays par des structures implantées aussi bien en éducation aux médias que dans la recherche :

- En Belgique : le groupe de recherche en médiation des savoirs, département de communication de l'Université catholique de Louvain,

- En Espagne: l'Universidad de Granada et l'Universidad de Huelva

- En France : le Clemi, centre de liaison de l'enseignement et des moyens d'information relevant du ministère de la Jeunesse, de l'Education nationale et de la Recherche,

- En Italie : l’Università cattolica del Sacro Cuore di Milano,

- Au Portugal : l’Universidade de Coimbra,

- Au Québec: l'Université de Sherbrooke et de l'Université de Montréal,

- En Suisse : le Centre d'éducation aux médias et aux technologies de l'information et de la communication (Cemtic).

L'enquête a été effectuée dans les institutions d'enseignement, auprès d'un échantillon total de 3326 jeunes issus de 7 pays. Pour chaque pays, elle s'est effectuée dans deux villes, dans deux écoles par ville et, dans chacune des écoles, auprès des cinq niveaux du secondaire ( élèves entre 12 et 18 ans).

Les données ont été recueillies selon deux modalités : le passage d'un questionnaire à l'ensemble de l'échantillon, puis des entretiens individuels avec un sous-échantillon. La dimension quantitative de l'analyse a permis de dresser un portrait des jeunes dans leur relation à Internet sur le plan statistique en termes d'accès, de fréquence d'utilisation, de types d'usage, de modes d'utilisation, d'opinions, etc. La dimension qualitative a permis de croiser et de vérifier les données, d'approfondir la variété des usages et des représentations d'Internet chez les jeunes et de saisir de façon plus fine leurs modes d'appropriation. 


\section{Quelle image les jeunes se font-ils d'Internet ?}

Dans tous les pays concernés par l'enquête, les adolescents qualifient très majoritairement Internet de « révolutionnaire ». Mais il s'agit pour eux plutôt d'une évolution radicale que d'une véritable révolution. Loin des discours souvent excessifs tant dans le panégyrique que dans l'anathème, les jeunes témoignent d'une modération qui s'accorde assez logiquement avec l'impression partagée qu'Internet, quoique reconnu technologiquement comme " révolutionnaire", s'intègre facilement au quotidien, sans perturbation majeure.

La grande majorité des jeunes ont une perception très positive d'Internet; ils le voient comme un outil pleinement utile et souhaitent sa généralisation : ceux qui y ont accès n'envisagent pas de s'en passer ; ceux qui n'en disposent pas y aspirent. Même chez ceux qui se disent, au départ, peu attirés par les nouvelles technologies et le monde des ordinateurs, Internet jouit d'un statut particulier. En raison de sa convivialité et de sa polyvalence, il peut répondre aux besoins et aux goûts de chacun.

Jugement majoritairement favorable et enthousiaste donc, mais non absolu ; loin d'être une panacée, Internet possède aussi ses limites aux yeux des adolescents. Si la communication leur apparaît généralement comme l'aspect le plus révolutionnaire d'Internet, certains émettent des réserves et lui reprochent l'anonymat et la dépersonnalisation : « J'ai des amis qui se sont rencontrés sur Internet, raconte Jacotte, ça marche bien sûr, mais c'est pas mon truc. Je préfère voir les gens en face de moi pour leur parler. »

L'image que les jeunes se font d'Internet semble étroitement liée aux discours sociaux véhiculés par les médias, les parents et les pairs. «Mes amis m'ont dit qu'Internet, c'est bien », dit Gertrudes ; « J'ai entendu parler des sites de pédophilie à la télévision », se souvient Steve. Les médias et les proches participent activement à la construction des représentations des jeunes, particulièrement quand leur pratique d'Internet est limitée. Plus l'usage devient fort et habituel, plus la force de ces représentations sociales et médiatiques se réduit. 


\section{Un moment de détente avant tout}

Les jeunes considèrent Internet avant tout comme un instrument de divertissement, même s'ils en reconnaissent l'intérêt pour apprendre ou pour exercer une profession. Cette perception, qui se retrouve dans tous les pays et qui semble s'être beaucoup amplifiée, recouvre autant une réelle pratique du loisir (celle du jeu ou du chat, par exemple) que celle d'activités plus complexes telles que la recherche d'informations, la rédaction de courriers électroniques... mais qu'Internet permet d'aborder de façon détendue. Si Internet apparaît comme un divertissement, c'est autant par ce qu' on peut y faire que par la manière plaisante de le faire.

Ils perçoivent aussi Internet comme un outil au service de leur envie de communiquer. Par sa rapidité, sa facilité, les possibilités d'aller à la rencontre de l'inconnu, la communication apparaît aux jeunes comme l'aspect le plus séduisant d'Internet. Parmi la diversité des actions possibles, la dimension communicative tient une place considérable dans la perception que ces jeunes ont du réseau, même si l'utilisation qu'ils en font reste souvent bien en deçà de ce qu'ils imaginent.

\section{Une information fiable... autant que dans les autres médias}

Les jeunes sont souvent convaincus qu'Internet recèle énormément de savoirs et d'informations - il faut toutefois savoir chercher. Ils comparent volontiers le Net à une mégabibliothèque, une encyclopédie sans limites et en constante expansion. Près des deux-tiers d'entre eux font confiance aux informations qui circulent sur le réseau. Mais pas une confiance aveugle : ils sont à peu près aussi nombreux à juger nécessaire un contrôle des sites. La plupart d'entre eux ont entendu parler de «dangers » liés à Internet, mais plus rares sont ceux qui y ont été confrontés directement. Le plus souvent, leur perception d'un danger provient des discours médiatiques et parentaux et la crainte qu'ils éprouvent est d'autant plus grande qu'ils connaissent mal Internet : moins les jeunes l'utilisent et moins ils lui font confiance.

Les jeunes n'interrogent pas spontanément la crédibilité et la fiabilité de l'information; pour eux, la question ne se pose pas plus pour Internet que pour les autres médias, exception faite des pages personnelles, lieu d'expression et d'opinions individuelles, dont ils reconnaissent la nature subjective. Pour le reste, ils s'en remettent au «bon sens ». Toutefois, le fait d'aborder avec eux cette question les conduit 
à douter, à hiérarchiser : certains estiment qu'il faudrait non pas interdire les sites, mais plutôt informer les jeunes (toujours les plus jeunes qu'eux), en évitant de mettre tous les «risques» sur le même niveau : «Les sites porno, ils font ce qu'ils veulent; mais racistes, il faudrait limiter parce que ça va à l'encontre des droits de l'homme », résume Cécile.

\section{Quelles pratiques les jeunes déclarent-ils ?}

La pratique diffère suivant l'âge et le sexe ; elle évolue surtout avec la connaissance d'Internet. Filles et garçons ont beaucoup de pratiques identiques : les unes et les autres sont très nombreux à déclarer visiter des sites Internet (91\%), utiliser les outils de recherche (91\%), chercher des images (87\%), envoyer du courrier électronique (72\%), et peu nombreux à laisser des commentaires sur les sites visités (30\%), répondre à des sondages ou à des questionnaires (26\%), créer des pages Web (25\%), participer à des groupes de discussion (25\%), cliquer sur des messages publicitaires $(21 \%)$, commander des produits (11\%). Cependant, les filles ont une prédilection plus marquée que les garçons pour le chat, et les garçons pour le téléchargement de musique et d'extraits vidéo.

Ces différences ont toutefois tendance à s'estomper avec le temps et la pratique régulière d'Internet. On y retrouve plutôt une variété d'usages de plus en plus individuels et personnalisés.

A travers les réponses des jeunes et l' analyse qu'en ont fait certaines équipes de recherche, il ressort quelques grands profils d'utilisateurs.

Ainsi l'équipe italienne a mis en évidence deux modèles dominants, qui montrent que les nouveaux médias et les nouvelles pratiques de lecture ne détruisent pas les plus anciennes, mais s'ajoutent et les modifient. D'un côté, certains jeunes reproduisent des comportements de consommation typiques de la lecture et de la culture du livre : ils ressentent le besoin d'imprimer les pages Web qu'ils ont trouvées, vont sur Internet comme ils iraient à la bibliothèque, disent préférer faire une recherche en bibliothèque parce que le bibliothécaire peut donner des conseils... De l'autre côté, d'autres jeunes reproduisent des comportements de consommation typiques de la télévision : ils visitent leur sites préférés comment s'ils consultaient les programmes des chaînes télévisuelles, entretiennent avec eux une relation de fidélité qui oppose 
à l'idée d'une navigation anarchique celle d'une navigation guidée par le besoin réel.

En France, l'enquête fait émerger deux grands groupes aux représentations et aux pratiques distinctes : d'un côté les usagers faibles ou nuls, les plus jeunes (moins de 15 ans) et plutôt les filles, de l'autre côté, les grands usagers, les plus âgés (15-17 ans) et plutôt les garçons.

Les jeunes du premier groupe ont découvert Internet récemment, à la maison ou à l'école, et l'utilisent peu. Leur pratique prend place essentiellement dans le cadre familial, et ressemble souvent à une balade sur le Web dépourvue d'objectifs très précis. Internet est pour eux un objet passionnant aux possibilités multiples, qui reste un peu mystérieux dans son fonctionnement. C'est dans ce groupe que se manifestent le plus souvent des réserves, parfois de l'inquiétude, devant un phénomène qu'ils ne maîtrisent pas, dont ils ont des échos parfois négatifs et dont ils mesurent mal l'incidence sur la vie quotidienne dans les années à venir.

Les jeunes du deuxième groupe, en particulier les plus de 15 ans, ont découvert Internet depuis plusieurs mois, voire deux ou trois ans, plutôt chez des amis. Ils se caractérisent avant tout par la multiplicité de leurs activités sur Internet : ils pratiquent aussi bien le Web que le chat, consultent, impriment, téléchargent, communiquent en fonction de leurs besoins et de leurs envies du moment, sont seuls ou avec des amis selon l'activité qu'ils pratiquent... Pour eux, Internet est à la fois un outil très utile, une source de loisir, une mine d'informations, un moyen de s'ouvrir sur le monde. C'est dans ce groupe que l'on trouve le plus de jeunes portant les jugements les plus positifs et les plus affirmés sur Internet - ils le trouvent révolutionnaire, facile, fiable, indispensable et réfutant le plus fort les arguments évoquant les dangers d'Internet.

\section{Une pratique plutôt individuelle, mais pas solitaire}

Les jeunes déclarent massivement (88\%) être seuls face à l'écran. Au Québec tout particulièrement, les parents sont très rarement conviés, les frères, sœurs ou amis sont tolérés comme une faveur qui leur serait accordée. Mais la pratique solitaire n'implique pas la solitude ni l'impression d'isolement.

Elle n'est pas non plus une pratique exclusive : un jeune sur deux déclare également aller sur Internet avec des amis. On ne peut donc pas dire que la pratique d'Internet couperait les jeunes de leur environnement amical et familial, elle est très loin d'apparaître comme une 
activité exclusivement solitaire. Les partenaires privilégiés des jeunes Français, par exemple, diffèrent selon leur degré de pratique : les petits utilisateurs choisissent de préférence leur entourage familial, les utilisateurs plus confirmés s'associent à leurs amis, qui prennent une place de plus en plus importante avec l'augmentation de la pratique. Au total, ce qui est marquant en France, c'est que plus on utilise Internet et plus on multiplie les cas de figure.

Les discussions sur Internet se développent majoritairement entre pairs aux dépens des relations "intergénérationnelles". En Belgique par exemple, la discussion avec les parents semble fortement liée à la culture familiale: les milieux familiaux soucieux d'éducation valorisent le débat à propos d'Internet et donnent aux jeunes des occasions de partager les opinions. Au contraire, les milieux socio-économiques modestes sont aussi ceux où les échanges et les débats sur Internet sont les moins fréquents et les moins valorisés.

Fait remarquable, c'est l'usage d'Internet qui en provoque l'apprentissage. Pour beaucoup de jeunes, les entretiens révèlent le rôle important joué par les pairs. Il s'agit d'un apprentissage par identification. "Mon ami me raconte ce qu'il fait et je le fais aussi”, tel pourrait être le paradigme de l'apprentissage d'Internet. L'identification au camarade semble pousser le jeune à tenter ses expériences et, finalement, à les réussir.

\section{Une tendance à tisser de " petites toiles personnelles"}

L'activité dominante de ces jeunes consiste beaucoup plus à revisiter des terrains connus qu'à tenter l'exploration de nouvelles parties du réseau. Chacun tisse sa petite toile, ratisse les mêmes sites connus, chatte avec les proches, explore peu de nouveaux domaines. A une exception près, la possibilité de se faire de nouveaux amis : en Italie, en France et au Québec, quatre jeunes sur dix déclarent s'être fait des amis sur Internet, alors qu'ils sont au moins les trois quarts à l'affirmer au Portugal et en Espagne.

Les jeunes ont peu la mémoire des sites visités et de leurs pérégrinations sur le Net. Ils passent d'un site à un autre sans souci du " chemin " pour s'y rendre. La très grande majorité a une idée plutôt vague de l'architecture de l'ensemble ; les jeunes naviguent " sans carte " et sans crainte de se perdre. Ainsi Franz « clique au hasard» sans trop savoir ce qu'il va trouver derrière le lien, parce qu'il pense que « c'est toujours sur le même sujet». Pourtant, «ça arrive souvent que ce ne soit pas 
sur le même sujet». Chez les petits utilisateurs, le surf renvoie à une consommation instantanée, éphémère et sans trace.

Pour la majorité des adolescents, Internet est loin d'être un lieu d'exploration et d'exploitation des ressources alternatives à la culture commerciale. Ils fréquentent peu les sites personnels, communautaires ou associatifs. S'ils restent amateurs de sites liés à leurs passe-temps favoris (musique, cinéma, jeux), ils se contentent généralement de prolonger sur Internet les relations qu'ils entretiennent avec des entreprises phares de l'industrie du divertissement largement sponsorisées par les grandes marques de vêtements et d'équipements sportifs (production musicale, cinéma, jeux vidéo, mode, sport professionnel, y compris les grandes chaînes de télévision et de radio). Il s'agit davantage d'une fréquentation passive plutôt que d'une consommation raisonnée qui les laisse peu enthousiastes.

On est souvent très loin de l'image du jeune internaute qui, grâce au branchement Internet à la maison, communique avec des correspondants du monde entier ou passe son temps à explorer de nouveaux domaines de connaissance. Dans la métaphore de la navigation chère aux internautes, les jeunes se laissent voir davantage comme des caboteurs que comme des marins d'horizons lointains. En dépit des aspirations de liberté, l'exploration déclarée d'Internet reste, somme toute, prudente et progressive.

Cette attitude, à première vue décevante, peut être interprétée comme une sorte d'auto éducation aux médias, consistant à observer ce que l'on connaît déjà à travers sa représentation véhiculée par une nouvelle technologie, afin de saisir l'apport spécifique de cette nouvelle technologie. Une attitude analogue apparaît chez les jeunes qui expérimentent le courrier électronique ou le chat en échangeant des messages avec leurs camarades de classe. Une telle méthode serait un moyen de se préparer à affronter l'inconnu en explorant préalablement le connu.

\section{C'est surtout à la maison que ça se passe}

L'élément le plus déterminant des relations entre l'adolescent et Internet est incontestablement la présence ou non d'un branchement à la maison. S'il y a un branchement à la maison, le jeune l'utilise. Cet accès lui permet d'utiliser Internet beaucoup plus souvent et plus régulièrement; la pratique est plus soutenue et plus sophistiquée. Les conditions mêmes de cet accès plus libre, plus autonome, plus individuel, diffèrent notablement de ce qu'offre le milieu scolaire. 
Internet se niche alors sans bouleversement dans les usages médiatiques des jeunes. Un peu moins de télévision et de vidéo, mais plus de musique, la part de la lecture et des sorties restant presque inchangée dans les déclarations des jeunes... « Internet, ça ne change rien à la télé, explique Steve. Je fais les deux en même temps. Dès que je me mets sur l'ordinateur, j'aime bien que la télé soit allumée. » Le plaisir de naviguer ne remplace pas les plaisirs différents que procurent la télévision, le cinéma ou la lecture. Internet n'occupe pas la place des autres médias, il s'y ajoute et les complète. Qui plus est, Internet fait plutôt bon ménage avec la musique : on peut l'écouter pendant qu'on navigue, on peut la télécharger.

\section{Internet à l'école : appropriation en cours}

C'est à l'école que plus de la moitié des jeunes ont découvert Internet. Mais, au-delà de ce rôle d'initiation, l'intégration d'Internet dans les pratiques pédagogiques et comme objet d'étude (par opposition à outil d'enseignement) est encore très irrégulière et généralement très faible. Malgré les investissements consentis et hormis les écoles dont l'informatique est la spécialité, la présence active d'Internet à l'école est souvent tributaire d'un enseignant engagé et convaincu. Appropriation en outre limitée, car les stratégies d'apprentissage développées à l'école autour d'Internet semblent, aux yeux des jeunes, confiner ces approches au milieu scolaire exclusivement, disjointes en cela du champ des pratiques extra-scolaires et surtout des pratiques spécifiquement mues par le plaisir. En ce sens l'enseignement ne contribue pas à aider les jeunes à comprendre leurs expériences ludiques.

A la maison, on a plus facilement la liberté d'accéder à Internet quand on veut et pour y faire ce qu'on veut; à l'école, Internet n'est accessible qu'à certaines heures pour y mener des activités bien précises et encadrées (recherche documentaire, construction de pages Web) alors que d'autres sont le plus souvent interdites (chat, téléchargement, jeux). Deux contextes d'usage différents, qui s'ignorent plus qu'ils ne se complètent et appellent à de nouvelles réflexions sur la nécessaire complémentarité d'Internet à l'école, par rapport à l'importance des usages domestiques.

Les jeunes souhaitent tous une présence renforcée d'Internet à l'école, autant comme outil d'apprentissage que parce qu'ils estiment 
essentiel d'en bien connaître le mode d'emploi, condition nécessaire à leur future intégration professionnelle. Les adolescents sont convaincus qu'une grande part de leur acquisition du savoir peut passer par Internet, à l'école ou ailleurs. En revanche, ils sont également très conscients du fait qu'Internet ne peut remplacer physiquement l'école dans sa structure classique (classe, professeur et tableau); pour eux, l'école est une occasion de socialisation que la virtualité du Net ne peut remplacer.

\section{Pas de portrait type du jeune internaute}

On le voit, les conditions et les lieux d'accès et de pratiques (le fait que le jeune dispose ou non d'un branchement chez lui ; qu'il ait l'occasion d'une pratique plus ou moins intensive à l'école) engendrent des approches d'Internet très distinctes. Le sexe, l'âge, le niveau de pratique et la familiarisation à Internet sont autant de variables qui influencent les modes d'usage, les objectifs recherchés et la manière de les atteindre. On constate que plus les jeunes pratiquent et plus leur usage se diversifie.

Une chose est claire : avec l'expérience, les profils des usagers se diversifient, même si des constantes restent sensibles. Toutefois, cette apparente diversité de se développe pas au hasard. En Belgique, où l'opposition socioéconomique entre les écoles présentes dans l'échantillon analysé est forte, nous avons pu observer combien non seulement l'accès, mais aussi la capacité critique et de réflexion des élèves, visà-vis d'Internet, étaient inégaux, et liés au niveau socioéconomique. Il y a là potentiellement un risque de dualisation, voire d'exclusion d'une partie moins favorisée de la population. Même les jeunes en sont conscients, puisqu'ils se révèlent assez sensibles au danger d'exclusion pour celui qui sera dépourvu d'Internet ou inapte à l'utiliser.

\section{Pistes pour la recherche}

Admettons-le. Outre la nécessaire actualisation de ces données, un certain nombre de questions ont été à peine effleurées et beaucoup de nouvelles interrogations ont surgi de l'enquête. Elles touchent principalement la manière dont les jeunes "apprennent Internet", évoluent 
dans leurs pratiques et développent une vision personnelle et critique de celui-ci. On peut également y ajouter des questions plus ponctuelles touchant, par exemple, la conception que les jeunes se font d'une activité documentaire et la place du plaisir dans cette activité, la notion de risque, l'identification des interlocuteurs à travers Internet, les apprentissages spécifiques, etc.

Il semble, aux yeux de l'équipe internationale qui s'est constituée, qu'une perspective plus féconde que la première consisterait en une recherche appliquée sur les usages que les jeunes ont d'Internet et des nouvelles technologies de l'information et de la communication incluant les technologies mobiles. Ces premiers résultats, qui éclairent la situation en l'an 2000, pourraient constituer une base de référence tant dans les objectifs que dans la démarche de recherche.

La recherche confirme la possibilité et l'intérêt de fonder une éducation aux médias sur l'expérience et les embryons de questionnement que construisent spontanément les jeunes, puisqu'ils découvrent Internet par leurs expériences. A ce titre, la présente recherche vient assurément apporter un matériau consistant. Une seconde perspective d'action éducative se profile en direction d'une pédagogie d'accompagnement du changement. Internet n'est pas un fait figé. C'est avant tout une dynamique d'évolution dans la communication sociale. Il importe par conséquent de concevoir une pédagogie capable de rendre les jeunes aptes à vivre ce changement, tout en restant conscients, autonomes et libres. Dans ces domaines, l'école, la famille et la tierce éducation (mouvements de jeunesses, associatifs, voisinage...) ont incontestablement un rôle à jouer.

Plusieurs structures partenaires de la recherche ont, entre 2000 et aujourd'hui, poursuivi leurs investigations, affiné leurs questionnements, développé des outils méthodologiques permettant de recueillir des informations qualitatives auprès des parents, des enseignants et des enfants. Elles pourraient à l'avenir conduire une recherche appliquée en éducation au média Internet, impliquant activement les jeunes et les éducateurs, en particulier dans le cadre européen.

\section{Notes}

1 CLEMI, Centre de liaison entre l'école et les médias d'aujourd'hui, Ministère de l'Education nationale (France). 